\title{
Cluster Evolution in Thermal Decomposition of CL-20/TNT Co-crystal in Compressed State: A ReaxFF Reactive Molecular Dynamics Simulation Study
}

\author{
Yan $\mathrm{Li}^{1}{ }^{1}{ }^{\star}$, Wen-li $\mathrm{Yu}^{1}$, Huang Huang ${ }^{2}$, Min $\mathrm{Zhu}^{2}$ \\ ${ }^{1}$ Rocket Force University of Engineering, Xi' an 710025, China. \\ ${ }^{2}$ Naval University of Engineering, Wuhan 430033, China.
}

\begin{abstract}
The thermal decomposition process of CL-20/TNT co-crystal in different compression states is simulated by ReaxFF reactive molecular dynamics method. Co-crystals models with different degrees of compression are obtained under four pressures of atmospheric pressure, 4.6GPa, 9.2GPa and 18.4GPa. The potential energy evolution, product formation and cluster evolution in the process are analyzed. We find that the formation of clusters is the key to the decomposition process. When the system is compressed, there will be more clusters which can bind the free atoms, resulting in the reaction to be inhibited. Other evolution information conforms this.
\end{abstract}

Key Words: ReaxFF force field; molecular dynamics; co-crystal of CL-20/TNT; thermal decomposition

In the process of initiation, the explosive will

\section{Introduction}

With the continuous evolution of modern war, there are higher requirements for weapon systems. Explosives are a very important components of weapons. Their characteristics determine the comprehensive performance of weapon systems to a great extent. Therefore, researchers in related fields have been pursuing to develop explosives that more meet the needs of modern war. However, there is a contradiction between the energy level and safety of explosives. How to deal with this contradiction has become the focus of attention in recent years. Co-crystallization is one of the effective ways to reconcile this contradiction. At present, many kinds of co-crystals explosives have been synthesized based on CL-20, the most representative of which is CL-20/TNT ${ }^{1-6}$. The two components have very distinct characteristics. CL-20 has excellent energy density, but its sensitivity is relatively high, which greatly limits the wide application of the explosive. TNT has low price and low sensitivity, but its energy density is relatively low compared with new explosives. Using co-crystallization strategy to fuse the two explosives from the crystal level, the co-crystals explosives with both energy level and safety can be obtained. The experimental results show that the detonation velocity of CL-20/TNT is $8.6 \mathrm{~km} \cdot \mathrm{s}^{-1}$ and its detonation pressure is $35 \mathrm{GPa}^{1}$. be compressed by the shock wave, and then ignited. Therefore, it is of great significance to study the thermal response of explosives in different compressed states. ROM et al. studied the thermal decomposition process of liquid TNT with different densities. The results show that for low-density TNT, the fracture of $\mathrm{C}-\mathrm{N}$ bond is the main initial reaction path, while for high-density TNT, dimers and small gas molecules are easier to form ${ }^{7}$. Wang studied the effect of density on thermal decomposition mechanism of $\varepsilon-C L-20$. The results show that that the frequency of $\mathrm{N}-\mathrm{NO}_{2}$ bond breakage decreases at high density ${ }^{8}$. Different compression states have certain effects on the thermal decomposition process of explosives.

For co-crystals explosives, there are more than two components in one cell, and the internal structures are more complex. In the process of thermal decomposition, the components affect each other, which may be quite different from that of simple explosives. Liu's research shows that TNT has a certain inhibitory effect on CL-20 decomposition in the process of CL-20/TNT co-crystals decomposition ${ }^{9}$. Yang studied the thermal sensitivity of CL-20/DNB co-crystals. The results show that the thermal sensitivity of CL20/DNB is lower than that of CL-20/TNT and CL-20 single crystal, but higher than that of DNB 
single crystal ${ }^{10}$. Miaoanalyzed the products, clusters and reaction paths during the thermal decomposition of CL-20/DNB ${ }^{11}$. In addition, there are some studies on the thermal decomposition process of CL-20 based co-crystals ${ }^{12-14}$.

At present, there are relatively few studies on the effects of different compressed states on the thermal decomposition process of co-crystals explosives, especially for cluster analysis. In this paper, ReaxFF reactive molecular dynamics simulation is used to simulate the thermal decomposition process of CL-20/TNT co-crystals in different compression states. The potential energy evolution, products formation and clusters evolution are analyzed. The effects of compression state on the thermal decomposition process of co-crystals are revealed from molecular scales.

\section{Methodology}

The CL-20/TNT co-crystals cell data used in this paper is derived from X-ray crystal structure ${ }^{1}$. The initial single crystal cell contains 8 CL-20 molecules and 8 TNT molecules. Based on this, the single crystal cell is expanded to $3 \times 2 \times 1$ supercell, which contains 64 CL-20 molecules and 64 TNT molecules, totaling 3648 atoms.

Firstly, the structure of the supercell is optimized to obtain the structure with the lowest energy. Then, the NVT ensemble is used for 10ps relaxation at $298 \mathrm{~K}$, berendsen temperature controller is selected for temperature control. In order to obtain the structure at atmospheric pressure, the NPT ensemble is used for $15 \mathrm{ps}$ relaxation at $298 \mathrm{~K}$ and $0 \mathrm{GPa}$, the Nosé-Hoover method is selected for temperature and pressure control. The co-crystals structure of CL-20/TNT at room temperature and pressure is obtained, the density is $1.89 \mathrm{~g} \cdot \mathrm{cm}^{-3}$. Using a similar method, the temperature is unchanged, and the pressures are adjusted to 4.6, 9.2 and 18.4GPa. The architectures in different compressed states are obtained, and the densities are $2.15,2.32$ and $2.53 \mathrm{~g} \cdot \mathrm{cm}^{-3}$ respectively. Finally, the NVT molecular dynamics simulations are performed at $2000 \mathrm{~K}$ and $3000 \mathrm{~K}$ to the systems in the four compression states respectively. The berendsen temperature controller is used as temperature control. There are 8 simulations. The time step is set to $0.1 \mathrm{fs}$ and the heating time is set to 100ps. All simulations use Lammps package ${ }^{15}$.

\section{Results and Discussions}

\subsection{Evolution of potential Energy and Species}

The system potential energy can reflect the process of explosive thermal decomposition to a certain extent. Fig. 1 shows the system potential energy evolution of CL-20/TNT co-crystals in different compression states at $2000 \mathrm{~K}$ and $3000 \mathrm{~K}$, respectively. It can be seen that in each state, the system potential energy first experience a short rapid increase, indicating that the system just began to heat and absorb energy from the outside. This time is also called induction period. Then, the potential energy begins to decrease, and the system releases energy. At this stage, the explosive gradually undergoes thermal decomposition reaction, releasing a large amount of heat energy, and the energy contained in the system decreases. Within 100ps and at $2000 \mathrm{~K}$, the potential energy of the system continues to decline, indicating that the thermal decomposition reaction of explosive still continues at a certain rate. At the temperature of $3000 \mathrm{~K}$, the system potential energy gradually tends to be stable after a rapid decline, indicating that the thermal decomposition reaction of explosive in the early stage at this temperature is more intense and the degree of thermal decomposition is high. When the reaction proceeds to about $40 \mathrm{ps}$, the exothermic process is basically completed.

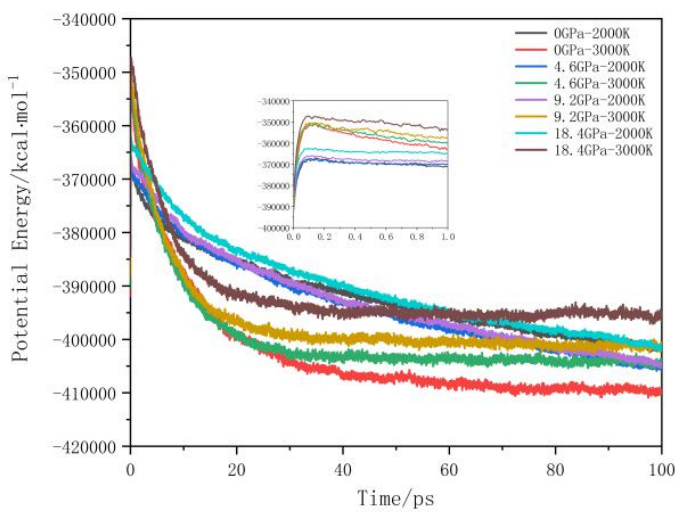

Fig. 1 Evolution of Potential Energy

The thermal decomposition of CL-20/TNT 
co-crystals is accompanied by the disassembly of explosive molecules into small molecules. The more the relative number of small molecules, the higher the degree of system decomposition. The degree of decomposition can be defined by the ratios of the numbers of molecules to the number of all atoms in the system, using $\gamma$ to express. For example, before thermal decomposition, the total number of molecules in the system is 128 (64 CL-20 molecules and 64 TNT molecules), and the total number of atoms in the system is 3648 , then $\gamma$ approximately equal to 0.035 . Fig. 2 shows the $\gamma$ evolution of CL-20/TNT co-crystals in different compression states at $2000 \mathrm{~K}$ and $3000 \mathrm{~K}$. At the temperature of $2000 \mathrm{~K}, \gamma$ increases steadily with the progress of the reaction; At the temperature of $3000 \mathrm{~K}, \gamma$ increases rapidly in the first $30 \mathrm{ps}$ and then tends to be stable. This shows that at the temperature of $2000 \mathrm{~K}$ the decomposition progress of the system is relatively slow and the reaction continues at a certain rate within $100 \mathrm{ps}$. At the temperature of $3000 \mathrm{~K}$, the reaction is relatively violent in the early stage, and the decomposition degree increases rapidly. When the decomposition reaches a certain degree, it tends to be stable. This is consistent with the reaction of potential energy evolution process. In addition, we can clearly find that compression will reduce the degree of system decomposition.

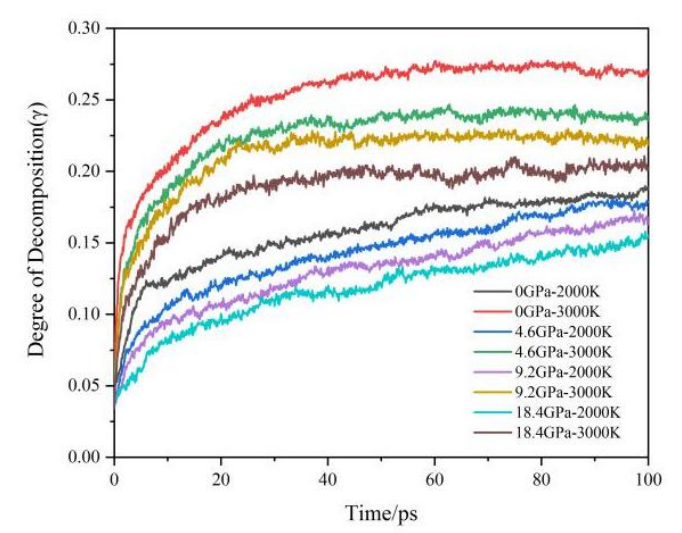

Fig. 2 Evolution of $\gamma$

\subsection{Products Analysis}

Firstly, the decomposition of reactants CL-20 and TNT in eutectic is analyzed. Fig. 3 shows the change of CL-20 molecular numbers under different conditions within $0-1$ ps. Fig. 4 shows the change of TNT molecular numbers under different conditions within $0-2 \mathrm{ps}$. It can be observed that temperature has a great influence on the decomposition rate of reactants CL-20 and TNT. At the temperature of $3000 \mathrm{~K}$, both CL-20 and TNT can decompose rapidly and thoroughly. At the temperature of $2000 \mathrm{~K}, \mathrm{CL}-20$ can decompose completely within $1 \mathrm{ps}$, but the overall decomposition rate is significantly slower than that at $3000 \mathrm{~K}$. There is still a part of TNT not decomposed. In addition, different compression states have little effect on the decomposition rate of reactants, and TNT is more difficult to decompose than CL-20.

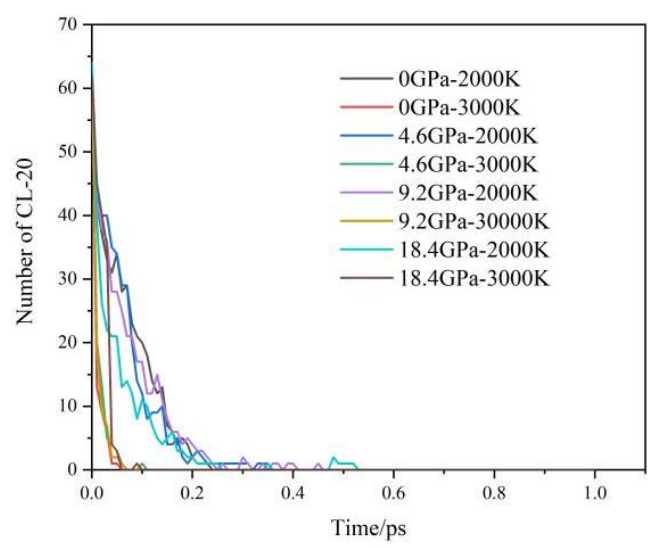

Fig. 3 Evolution of Number of CL-20

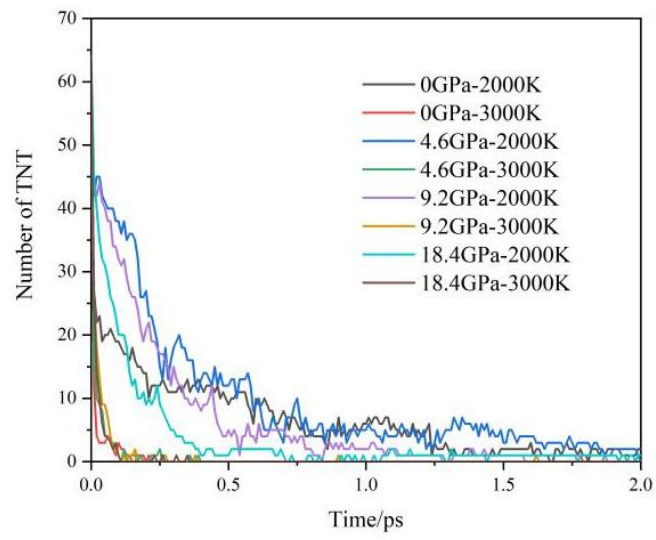

Fig. 4 Evolution of Number of TNT

According to previous scholars' research, the products of CL-20/TNT co-crystals thermal decomposition are mainly composed of intermediate products and final products. The most important intermediate product is $\mathrm{NO}_{2}$. The main final products are $\mathrm{CO}_{2}, \mathrm{H}_{2} \mathrm{O}$ and $\mathrm{N}_{2}$. Fig. 5 shows the change of $\mathrm{NO}_{2}$ quantity in the system with time. A 
large number of studies show that the thermal decomposition reaction of many nitroexplosives starts from the generation of $\mathrm{NO}_{2}$, and the change of $\mathrm{NO}_{2}$ quantity directly reflects the process of thermal decomposition. At $2000 \mathrm{~K}$, a certain amount of $\mathrm{NO}_{2}$ exist in the system for a long time, while at $3000 \mathrm{~K}$, $\mathrm{NO}_{2}$ only exist in the system for the first 50ps. This shows that at the temperature of $2000 \mathrm{~K}$ the thermal decomposition reaction process of co-crystals explosive is slow, the reaction lasts for a long time. At the temperature of $3000 \mathrm{~K}$, the reaction is relatively rapid, the large-scale decomposition process has been completed at 50ps. At the same temperature, the amount of $\mathrm{NO}_{2}$ in the thermal decomposition process of co-crystals in atmospheric pressure state is significantly more than that in compression state, which shows that compression can inhibit the reaction.

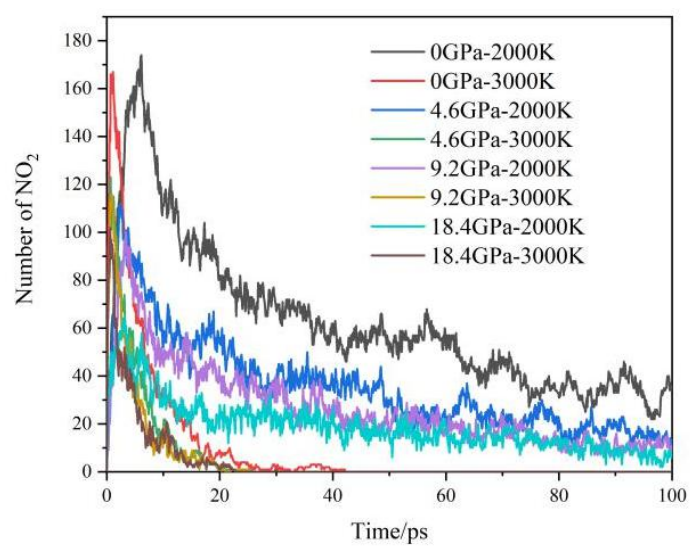

Fig. 5 Evolution of Number of $\mathrm{NO}_{2}$

Fig. 6 shows the evolution of the number of $\mathrm{CO}_{2}, \mathrm{H}_{2} \mathrm{O}$ and $\mathrm{N}_{2}$ in the system with time. At the temperature of $2000 \mathrm{~K}$, within $10 \mathrm{ps}$, the numbers of three main final products in systems with different compression states are still in the rising stage, that is, the reaction is not complete, and the change curves of the three products are similar. At the temperature of $3000 \mathrm{~K}$, the number of three main final products in systems with different compression states increased at a rapid rate at first, and then tended to be stable. In the steady state, the higher the degree of compression, the less the number of three products, that is, the less complete the reaction, which is consistent with the previous analysis conclusion on the decomposition degree of co-crystals. For $\mathrm{CO} 2$, the difference of number caused by temperature is large. At $2000 \mathrm{~K}$, the number of $\mathrm{CO}_{2}$ is in an upward trend and is less as a whole. At $3000 \mathrm{~K}$, the number of $\mathrm{CO}_{2}$ increases rapidly and reaches a stable level, with a large amount as a whole. This is mainly because when the temperature is high, a large number of carbon rings in the system decompose and free carbon atoms increase, resulting in more $\mathrm{CO}_{2}$. For $\mathrm{H}_{2} \mathrm{O}$ and $\mathrm{N}_{2}$, the difference of number caused by temperature is relatively small. At $100 \mathrm{ps}$, the number of $\mathrm{H}_{2} \mathrm{O}$ produced by some compressed systems at $2000 \mathrm{~K}$ is even more than that at $3000 \mathrm{~K}$. This is mainly because free oxygen atoms tend to generate $\mathrm{CO}_{2}$ with free carbon atoms at high temperature, while at low temperature, the number of free carbon atoms is less, while oxygen atoms and hydrogen atoms generate more $\mathrm{H}_{2} \mathrm{O}$.

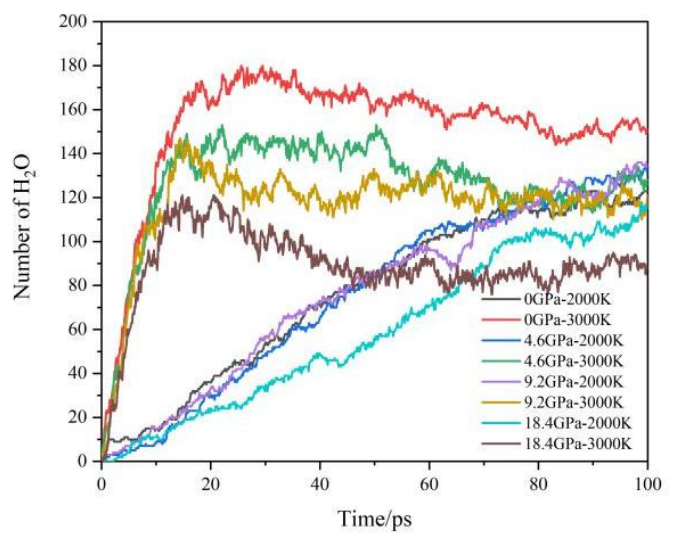

(a)

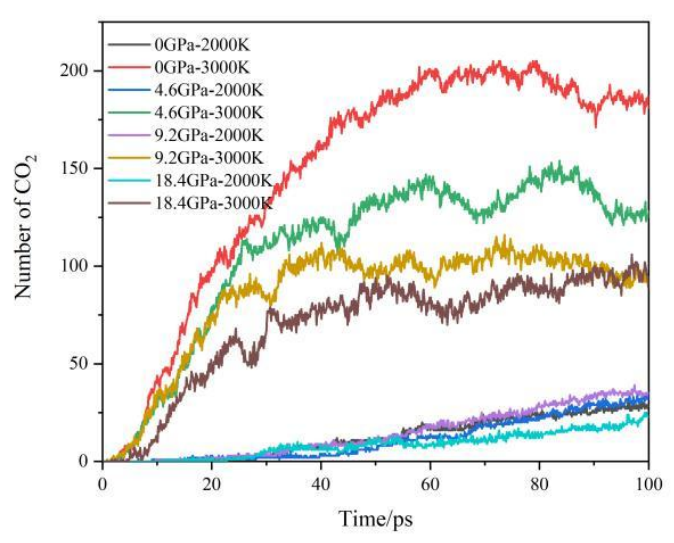

(b) 


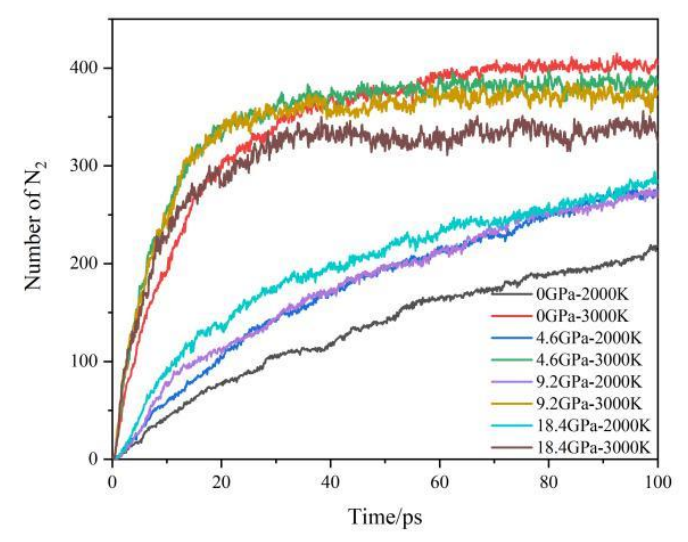

(c)

Fig. 6 Evolution of Number of

Main Final Products

\subsection{Evoliton of Clusters}

\subsubsection{Amount of Clusters}

As shown in Fig. 7, the number of clusters begin to increase rapidly at the beginning of heating. At $2000 \mathrm{~K}$, the number of clusters hovers between 5-10 under atmospheric pressure, 15-20 under 4.6 GPa compression, 26 under 9.2 GPa compression, and then hovered between 15-20. Under 18.4GPa compression, the number of clusters peak at 25 , then decrease gradually, stabilizing at about 8 . At $3000 \mathrm{~K}$, the number of clusters hovers around 5 at atmospheric pressure, and the numbers of clusters in the other three compressed states are close, hovering between 8-17. Comparing the evolution of the numbers of clusters in the reaction process of eutectic systems with different compression states at the same temperature, it is found that a certain compression will increase the number of clusters in the reaction process, but the degree of compression has little effect on the number of clusters. In addition, comparing the evolution of the number of clusters in the reflection process of the same compression state at different temperatures, it is found that when the temperature is higher, the number of clusters decreases slightly, indicating that the higher temperature leads the atoms in the system tend to exist in the state of small molecules. At the same time, it can also be observed from the figure that at $2000 \mathrm{~K}$, the number of clusters in the $18.4 \mathrm{GPa}$ compressed state in the second half of the heating process (50ps to 100ps) is slightly higher than that in the atmospheric pressure state, lower than that in other compressed states, which may be greatly related to the size of a single cluster.

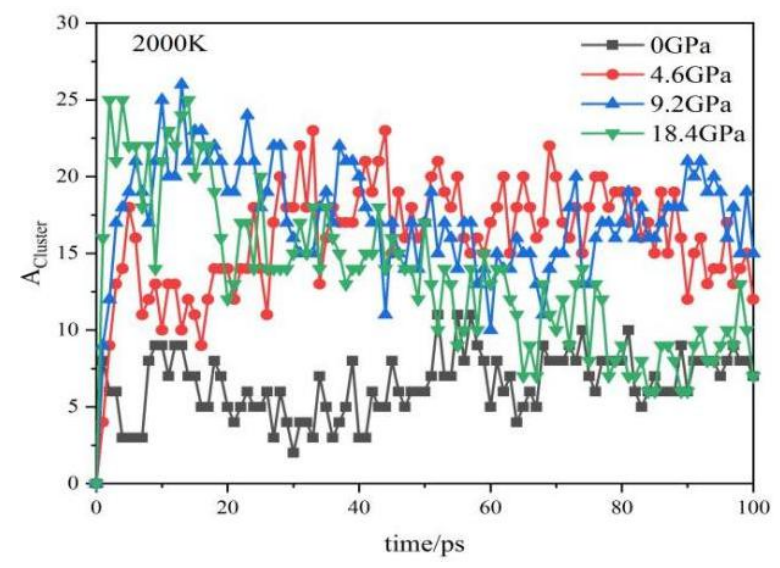

(a)

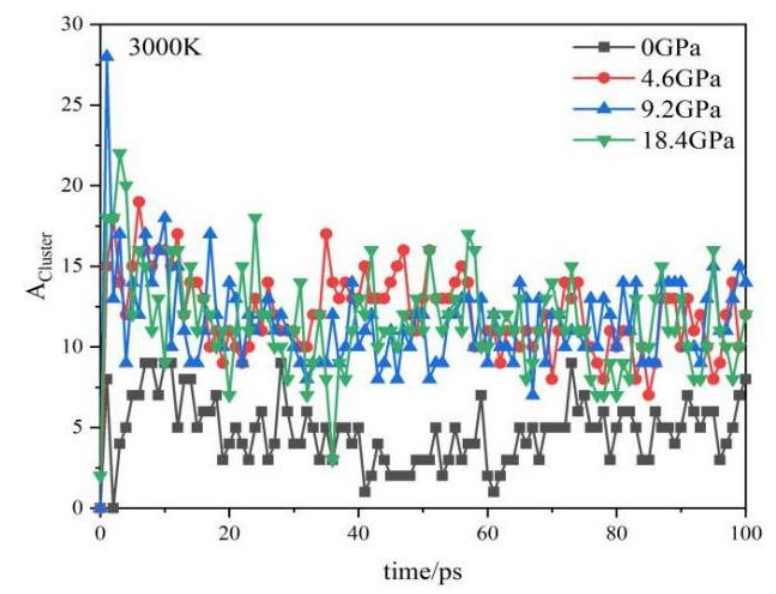

(b)

Fig. 7 Evolution of Number of Clusters

\subsubsection{Size of Clusters}

As shown in Figure 8, at the same temperature, with the increase of compression degree, the clusters in the system will gradually become larger. It can be inferred that the higher the degree of compression, inducing the distance between atoms smaller, and the collision probability between atoms increases greatly, making atoms more inclined to gather and form large clusters. Large clusters can inhibit the decomposition of reactants, that is, the higher the degree of compression, the lower the degree of decomposition of the system. This is consistent with the results of the decomposition degree change diagram under different conditions. Comparing the 
clusters size distribution under different temperatures in the same compression state, it can be seen that higher temperature will promote the decomposition, resulting in the size of cluster to be smaller in the system.

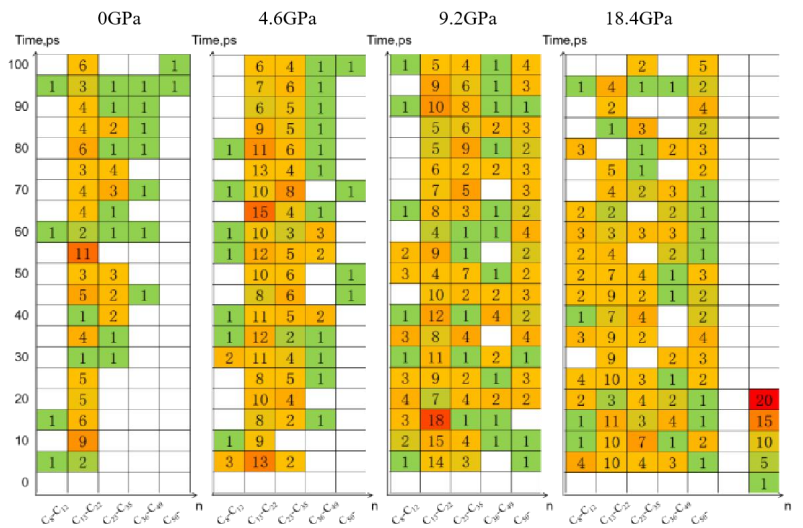

(a) $2000 \mathrm{~K}$

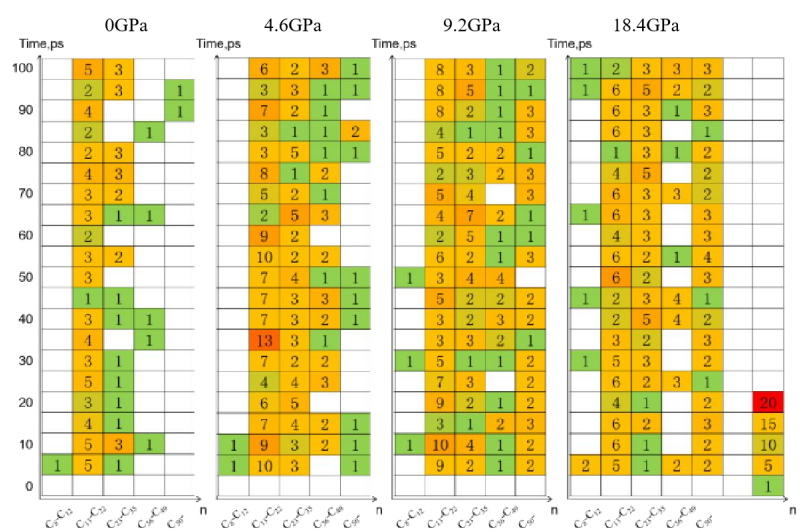

(b) $3000 \mathrm{~K}$

Fig. 8 Distribution of Clusters Size

Fig. 9 shows the change of mass ratios of clusters in the system. It can be observed that the higher the degree of compression leaded the higher mass ratios of clusters in the system. At $2000 \mathrm{~K}$, the mass ratio of clusters in atmospheric pressure system is about one tenth, and the mass proportion of clusters in $18.4 \mathrm{GPa}$ system with the highest degree of compression can reach one-half. Under the high degree compression, although there is little change in the number of clusters, the size of clusters increases significantly, so that more atoms are bound to large clusters, and the reaction is inhibited.

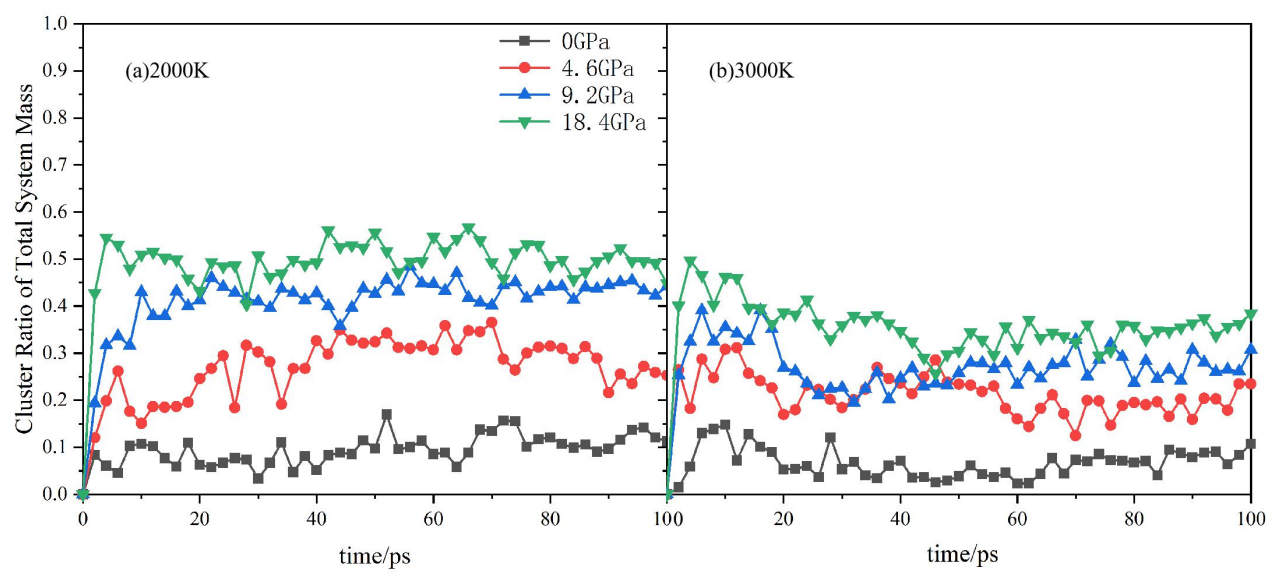

Fig. 9 Evolution of Clusters Mass Ratio

\section{Conclusion}

Using the ReaxFF reactive molecular dynamics simulation, we study the thermal decomposition of co-crystals CL-20/TNT in different compression states. The potential energy, species, products and clusters are analyzed. The results show that compression will reduce the distance between atoms in the system, increase the collision probability of atoms, and produce more clusters. Large clusters will bind atoms and inhibit the reaction of the system. All the information confirms this conclusion. With the compression, the variation range of potential energy decreases, the decomposition degree of system decreases and the 
products reduce. The clusters play an important role of thermal decomposition of co-crystals CL-20/TNT under compression.

\section{Author information}

Corresponding Author

E-mail: yacareft@163.com

\section{Declarations}

Funding: Not applicable

Conflicts of interest/Competing interests: Not

applicable

Availability of data and material: Not applicable

Code availability: Not applicable

Authors' contributions: Not applicable

\section{References}

(1) Bolton, O.; Matzger, A. J. Angew. Chem. Int. Ed. 2011, 50, 8960. doi: 10.1002/ange.201104164

(2) Bolton, O.; Simke, L. R.; Pagoria, P. F.; Matzger, A. J. Cryst. Growth Des. 2012, 12, 4311. doi: 10.1021/cg3010882

(3) Wang, Y.; Yang, Z.; Li, H.; Zhou, X.; Zhang, Q.; Wang, J.; Liu, Y. Propell. Explos. Pyrotech. 2014, 39, 590.

doi: 10.1002/prep.201300146

(4) Xu, H.; Duan, X.; Li, H.; Pei, C. RSC Adv. 2015, 5, 95764. doi: 10.1039/C5RA17578J

(5) Duan, B.; Shu, Y.; Liu, N.; Wang, B.; Lu, X.; Lu, Y. CrystEngComm 2018, 20, 5790. doi: 10.1039/C8CE01132J

(6) Huang, C.; Xu, J.; Tian, X.; Liu, J.; Pan, L.; Yang, Z.; Nie, F. Cryst. Growth Des. 2018, 18, 2121. doi: 10.1021/acs.cgd.7b01568

(7) Naomi, R.; Barak, H.; Yehuda, Z.; David, Furman.; Sergey V.; William A.; Ronnie, K. J. Phys. Chem. C 2013, 117, 21043. doi:10.1021/jp404907b

(8) Wang, F.; Chen, L.; Geng, D.; Lu, J.; Wu, J. Phys. Chem. Chem. Phys. 2018,20, 22600 doi:10.1039/C8CP03010C

(9) Liu, H.; Yang, Z.; He, Y. Chin J Explos Propellants 2017, 40, 14. doi: 10.14077/j.issn.1007-7812.2017.01.003

(10) Yang, Z.; Xue, Y.; He, Y. Acta Chim. Sinica 2016, 74, 612 doi: $10.6023 / \mathrm{a} 16030141$

(11) Miao, R.; Liu, W.; Wang, J.; Kang, Z.; Yang, L.; Jing, X.; Fu, Y.; Liu, Y. Chin J Energ Mater 2016, 24, 111 doi: 10.11943/j.issn.1006-9941.2016.02.001

(12) Xue, X.; Ma, Y.; Zeng, Q.; Zhang, C. J. Phys. Chem. C 2017, 121, 4899 doi: 10.1021/acs.jpcc.7b00698
(13) Ren, C.; Liu, H.; Li, X.; Guo, L. Phys. Chem. Chem. Phys. 2020, 22, 2827 doi: $10.1039 / \mathrm{C} 9 \mathrm{CP} 06102 \mathrm{~A}$

(14) Guo, D.; An, Q.; Zybin, S.; Goddard, W.; Huang, F.; Tang, B. J. Mater. Chem. A 2015, 3, 5409 doi: 10.1039/C4TA06858K

(15) https://www.lammps.org/ (accessed Jul. 30, 2021) 